\title{
Modeling the impact of a molten metal droplet on a solid surface using variable interfacial thermal contact resistance
}

\author{
Minxia Xue $\cdot$ Yoav Heichal $\cdot$ Sanjeev Chandra $\cdot$ \\ Javad Mostaghimi
}

Received: 7 July 2006/ Accepted: 11 October 2006/Published online: 11 November 2006

(C) Springer Science+Business Media, LLC 2006

\begin{abstract}
An analytical model of the true area of contact between molten metal and a rough, solid surface has been used to calculate thermal contact resistance and to predict how it changes with surface roughness, substrate thermal properties and contact pressure. This analytical model was incorporated into a three-dimensional, time-dependent numerical model of free-surface flows and heat transfer. It was used to simulate impact, spreading and solidification of molten metal droplets on a solid surface while calculating contact resistance distributions at the liquid-solid interface. Simulations were done of the impact of $4 \mathrm{~mm}$ diameter molten aluminum alloy droplets and $50 \mu \mathrm{m}$ diameter plasma sprayed nickel particles on steel plates. Predicted splat shapes were compared with photographs taken in experiments and simulated substrate temperature variation during droplet impact was compared with measurements.
\end{abstract}

\section{Introduction}

Spray coating and spray forming are processes in which droplets of molten metal or ceramic impinge on a surface, solidify and coalesce with each other to form solid deposits. The physical properties of the solid

M. Xue $(\bowtie)$ · Y. Heichal · S. Chandra · J. Mostaghimi Department of Mechanical and Industrial Engineering, Center for Advanced Coating Technologies, University of Toronto, 5 King's College Road, Toronto, ON, Canada M5S 3G8

e-mail: mxue@mie.utoronto.ca material formed are very sensitive to the cooling rate of droplets. Droplets freeze as they impact and flatten, and the shapes of the final splats determine properties such as porosity, roughness and thickness $[1,2]$. The microstructure of solidified droplets also depends on how rapidly they solidify. Determining and controlling the cooling rate of impacting particles in spray processes is a problem with important industrial applications.

Bussmann et al. and Pasandideh-Fard et al. developed a three-dimensional model of liquid droplet impact and solidification, which could be used to simulate droplets impinging on a solid surface [3, 4]. In such models it is necessary to specify a boundary condition for the spreading liquid at the dropletsubstrate interface. Assuming perfect liquid-solid impact, so that there is no discontinuity in the temperature distribution at the interface, is unrealistic. When a droplet of molten metal hits a rough, solid surface, air is trapped in crevices at the liquid-solid interface, creating an insulating layer whose value depends on surface finish, contact pressure and material properties. The temperature of the liquid $\left(T_{d}\right)$ therefore remains higher than that of the substrate $\left(T_{w}\right)$ driving a heat flux $\left(q^{\prime \prime}\right)$ across the interface. A "thermal contact resistance" $\left(R_{c}\right)$, conventionally used to describe the discontinuity for purposes of analysis, is defined as:

$R_{c}=\frac{T_{d}-T_{w}}{q^{\prime \prime}}$

Thermal contact resistance between two solid bodies has been a subject of study for many years and is reasonably well understood. Semi-empirical correlations exist that predict $R_{c}$ as a function of material properties, roughness and contact pressure [5]. However, heat transfer across a 
liquid-solid interface is not as well characterized. In the absence of any theoretical model to predict contact resistance several researchers have directly measured the cooling rates of molten metals deposited on colder substrates and calculated the resistance between the two from analytical or numerical models [6]. Differences in measurement techniques, material properties and experimental conditions have meant that reported values span several orders of magnitude.

Numerical models of molten metal droplet impact specify thermal contact resistance as an input to simulations. The absence of reliable data has meant that $R_{c}$ is often treated as a fitting parameter, whose value is adjusted to give the best agreement with observed droplet impact dynamics [4]. However, this approach limits the predictive capabilities of such models.

Qiu et al. proposed a simple correlation for the thermal contact conductance during the rapid contact solidification process [7]. By introducing this correlation into the numerical simulation a non-constant thermal contact resistance, which varied with time and position, was taken into consideration to simulate the spreading and solidification of a molten droplet on a substrate.

Heichal and Chandra used an analytical model of the deformation of a free liquid surface in contact with a rough solid surface to calculate the true area of contact between them, and thereby $R_{c}$ [8]. Thermal contact resistance was measured experimentally by recording surface temperature under impacting molten metal droplets and using a one-dimensional heat conduction model to calculate $R_{c}$. Experiments were done for a range of droplet and surface materials, surface roughness and impact velocities; predictions from the model were found to agree well with measured values of thermal contact resistance.

The objective of this paper was to demonstrate that the analytical model of Heichal and Chandra to predict thermal contact resistance [8] can be integrated in a numerical model of molten droplet impact and solidification. The value of thermal contact resistance will not be specified as an input: only the average surface roughness and thermophysical properties of the substrate have to be known. Calculated droplet shapes during impact were compared with photographs of molten aluminum alloy 380 landing on a steel surface and computed substrate temperature variation under impacting droplets with measured values.

\section{Experimental method}

Droplets of molten aluminum alloy 380 (Al 85.5\%, Si $8.5 \%, \mathrm{Cu} 4 \%, \mathrm{Fe} 2 \%$ ) were formed with a pneumatic droplet generator that consisted of a heated graphite chamber containing molten metal. Droplets $(4.0 \mathrm{~mm}$ in diameter) were forced out through a $2.0 \mathrm{~mm}$ diameter synthetic sapphire nozzle set in the bottom of the chamber by applying a rapid pulse of compressed nitrogen [9]. The droplet generator was supported on a frame with adjustable height, giving impact velocities of $1-3 \mathrm{~m} / \mathrm{s}$.

Test surfaces $(50.8 \times 50.8 \times 6.35 \mathrm{~mm}$ in size $)$ were made of H13 tool steel and mounted on a copper heater block whose temperature could be controlled. Test surfaces were polished on an electric belt-sander to produce average surface roughness $\left(R_{a}\right)$ between $5.0 \mu \mathrm{m}$ and $0.5 \mu \mathrm{m}$.

Photographs of impacting droplets were taken using a single shot flash photographic method [10]. A Nikon E3 digital camera was used to take a photograph of an impacting drop with a single $10 \mu$ s exposure flash. By varying the time delay between release of a droplet and triggering of the flash different stages of droplet impact were recorded and an entire impact sequence was reconstructed from a succession of such images.

Fast-response, thin-film thermocouples were used to measure surface temperature variation under impacting droplets. Heichal and Chandra have given a detailed description of the construction and operation of the thermocouples [8]. The conductive steel acted as one of the thermocouple materials. A $0.254 \mathrm{~mm}$ diameter Constantan wire was inserted through a $0.57 \mathrm{~mm}$ diameter hole in the substrate filled with ceramic cement that held the wire in place and insulated it electrically from the substrate. A thin conductive film of graphite was applied on the surface to form an electrical connection between the wire and surrounding steel. The voltage difference between the graphite film and a reference junction kept in an ice bath at $0{ }^{\circ} \mathrm{C}$ was calibrated as a function of the thermocouple temperature. Substrate temperature variation under an impacting droplet was measured by an array of thin film thermocouples spaced $1.15 \mathrm{~mm}$ apart. The time-varying signal from the thin film thermocouples was recorded by a data acquisition system.

\section{Model of droplet impact}

\section{Numerical model}

The numerical code used to model droplet impact solves equations of mass and momentum and energy discretized using a finite volume technique on a 3-D Eulerian structured grid. Details of the numerical model have been described in detail earlier [4]. The free surface of 
the droplet was modeled by defining the volume-of-fluid scalar $(f)$, as the fraction of a cell volume occupied by fluid, equal to one for a cell full of fluid, zero for an empty cell, and a fraction between zero and one for a free surface cell. The volume-of-fluid scalar is advected at a rate described by the equation

$\frac{\partial f}{\partial t}+(\vec{V} \cdot \vec{\nabla}) f=0$

where $\vec{V}$ is the velocity vector and $t$ the time. To identify and track the solid phase a second liquid volume fraction $(\lambda)$ is defined as a parameter whose value is equal to one in the liquid and zero in the solid and a fraction in cells at the liquid-solid interface. In locations where both liquid and solid phases exist, the liquid phase has volume fraction $\lambda$, and the solid phase $(1-\lambda)$, in which the liquid portion $(\lambda)$ is free to flow while the remaining portion $(1-\lambda)$ is frozen. To account for solidification the advection equation is modified to

$\frac{\partial f}{\partial t}+(\lambda \vec{V} \cdot \vec{\nabla}) f=0$

The method of solving conservation equations in cells with both liquid and solid is the same as those used for cells with no solid, except that solidified regions are treated as a liquid with zero velocity. Having defined the new volume fraction for solid cells, the conservation equations of mass and momentum are written as:

$\vec{\nabla} \cdot(\lambda \vec{V})=0$

$\frac{\partial(\lambda \vec{V})}{\partial t}+(\lambda \vec{V} \cdot \vec{\nabla}) \vec{V}=\frac{-\lambda}{\rho} \vec{\nabla} p+\lambda v \nabla^{2} \vec{V}+\frac{\lambda}{\rho} \overrightarrow{F_{b}}$

where $v$ is the kinematic viscosity, $\rho$ is the density, $p$ is the pressure, and $\vec{F}_{b}$ is any body force acting on the fluid. Surface tension was considered to be a component of the body force acting on the fluid free surface. Fluid flow was assumed to be Newtonian, laminar and incompressible. Any effect of the ambient air on the droplet evolution was neglected. Only normal stresses were assumed to act on the free surface. Laplace's equation was used to determine the pressure at the free surface.

The conservation of energy equation combined with the enthalpy-transforming model was used to model heat transfer [4]. Assuming that the phase change occurs at a single temperature, the temperature $(T)$ is related to enthalpy $\left(H_{e}\right)$ by

$T=T_{m}+\left(\gamma H_{e}+\phi\right) / k$ where $T_{m}$ is the melting point and $k$ is the thermal conductivity. Depending on the state of fluid, the coefficients $\gamma$ and $\phi$ can be defined as:

$\left\{\begin{array}{l}\gamma=k_{s} / C_{s} \text { and } \phi=0 H_{e} \leq 0(\text { solid phase) } \\ \gamma=0 \text { and } \phi=00<H_{e}<H_{f} \text { (liquid }- \text { solid interface) } \\ \gamma=k_{l} / C_{l} \text { and } \phi=-H_{f} k_{l} / C_{l} \quad H_{e} \geq H_{f} \text { (liquid phase) }\end{array}\right.$

where $C$ represents the specific heat, $H_{e}$ the enthalpy and $H_{f}$ the heat of fusion. The energy equation becomes:

$\rho \frac{\partial H_{e}}{\partial t}+\rho(\vec{V} \cdot \vec{\nabla}) H_{e}=\nabla^{2}\left(\gamma H_{e}\right)+\nabla^{2} \phi$

No-slip and no-penetration boundary condition were applied at solid surfaces. Liquid-solid contact angles were assumed to be $90^{\circ}$. Free surfaces of droplets and exposed portions of the substrate were assumed adiabatic.

Thermal contact resistance model

Mikic's model of thermal contact resistance between rough, solid surfaces pressed together was based on calculating the actual area of contact between surface asperities, rather than their nominal surface area [5]. The only mode of heat transfer was assumed to be conduction across these regions of contact, since convection and radiation across gaps are typically negligible. The contact resistance was a function of the contact pressure, surface roughness and hardness of the contacting solids.

Heichal and Chandra developed a similar theory to predict contact resistance between a liquid metal and a rough solid surface [8]. The rough surface was idealized as consisting of a series of half-cylinders placed next to each other (see Fig. 1), all having the same radius $\beta$, which proved to be a reasonable description of surfaces prepared by polishing with emery paper in one direction. Surface roughness $R_{a}$ is defined as the arithmetic average of the height of surface peaks above a hypothetical perfectly smooth plane. Figure 1 shows an idealized representation of liquid metal in contact with a rough solid substrate. Surface tension prevents the metal from penetrating into the valleys between surface peaks. For the ideal surface shown in Fig. 1 the average roughness is

$R_{a}=\frac{\int_{0}^{2 \beta} y(x) \mathrm{d} x}{2 \beta}=\frac{\int_{0}^{\pi} \beta^{2} \sin ^{2}(\psi) \mathrm{d} \psi}{2 \beta}=\frac{\pi \beta}{4}$ 


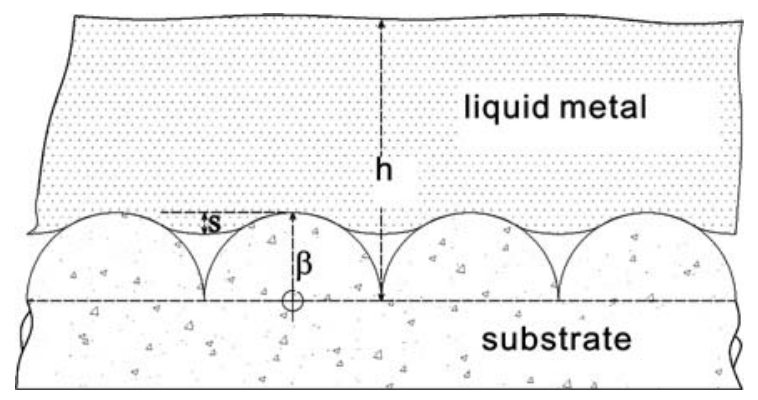

Fig. 1 Geometry of contact meniscus formed with spherical asperity

Thermal contact resistance is defined with respect to a plane passing through the base of surface asperities (see Fig. 1), whose temperature is $T_{w}$. Heat conduction ( $q$ ) from the liquid at temperature $T_{d}$ to this plane through a solid interface region of thermal conductivity $k_{w}$ is:

$q=\frac{k_{w}\left(T_{d}-T_{w}\right)}{R_{a}} \times A \times f_{A}$

where $A$ is the area of the reference plane and $f_{A}$ is the fraction of that area in contact with liquid metal. Combining Eq. (10) with the definition of thermal contact resistance equation (1) gives:

$R_{c}=R_{a} / k_{w} f_{A}$

For a perfectly smooth substrate $f_{A}=1$ and $R_{c}=R_{a} / k_{w}$. For a rough substrate we need to evaluate the true area of contact when a liquid metal column is pressed against it. Figure 1 shows the geometry of the interface, where a liquid layer of height $h$ is supported on a solid surface consisting of an array of semi-circular protrusions of radius $\beta$. Timsit derived a model to calculate the profile of the liquid meniscus by using variational methods to determine the shape that minimized the total potential and surface energy of the system [11]. To obtain an analytical solution it was assumed that surface asperities were much smaller than the liquid film thickness $(\beta<<h)$, that the liquid surface underwent only small deformation (i.e., $s<<\beta$ ) and the liquidsolid contact angle was $180^{\circ}$. Timsit demonstrated that the ratio of the true area of contact to the nominal surface area was:

$f_{A}=\frac{\beta h}{(\sigma / \rho g)+\beta h}$

where $g$ is the gravity acceleration and $\sigma$ is the surface tension of the liquid [11].
The height of the liquid column $h$ creates a hydrostatic head that presses the liquid down. It can be replaced by a local liquid pressure:

$p_{c}=\rho g h$

Substituting Eqs. (9) and (13) in Eq. (12) gives an expression for $f_{A}$ :

$f_{A}=\frac{p_{c}}{p_{c}+\pi \sigma /\left(4 R_{a}\right)}$

and by substituting Eq. (14) into Eq. (11), we can get an equation that allows us to calculate the thermal contact resistance of the interface region as a function of the contact pressure, material properties and average surface roughness:

$R_{c}=\frac{R_{a}\left[p_{c}+\pi \sigma /\left(4 R_{a}\right)\right]}{k_{w} p_{c}}$

In Eq. (15) surface roughness, $R_{a}$, was an input to the model. Liquid surface tension and the thermal conductivity of the substrate were each assumed to be a function of temperature, and their values at different temperatures were supplied in an input table (see Table 1). During simulations, the values of liquid surface tension and the thermal conductivity were updated with time by interpolation between the values given in Table 1, as a function of local temperatures. Aluminum alloy 380 does not have a sharp melting point, but a liquidus temperature of $593{ }^{\circ} \mathrm{C}$ and solidus temperature of $538{ }^{\circ} \mathrm{C}$. In simulations it was assumed that it behaved as a pure metal with a melting temperature of $570{ }^{\circ} \mathrm{C}$.

The pressure distribution in the liquid was calculated at each time step, and local interfacial thermal contact resistance values calculated from Eq. (15). As pressure increased, contact resistance decreased. It was assumed, though, that local contact resistance would not increase even if the local pressure was subsequently reduced. The physical reasoning is that once high pressure forces molten metal into substrate crevices, good contact is established that persists even if the local pressure decreases. Trials showed that this rule produced accurate simulations. Allowing the local contact resistance to fluctuate rapidly in response to rapid pressure changes produces numerical instabilities, and is probably physically unrealistic. For part of the interface where there was no fluid, meaning empty cells, the contact resistance of empty cells was set to $10^{10} \mathrm{~m}^{2} \mathrm{~K} / \mathrm{W}$, which led to negligible heat transfer.

The modified Navier-Stokes equations were solved on an Eulerian, rectangular, staggered mesh in a 3D 
Table 1 Properties of nickel, aluminum alloy, steel H13 and stainless steel

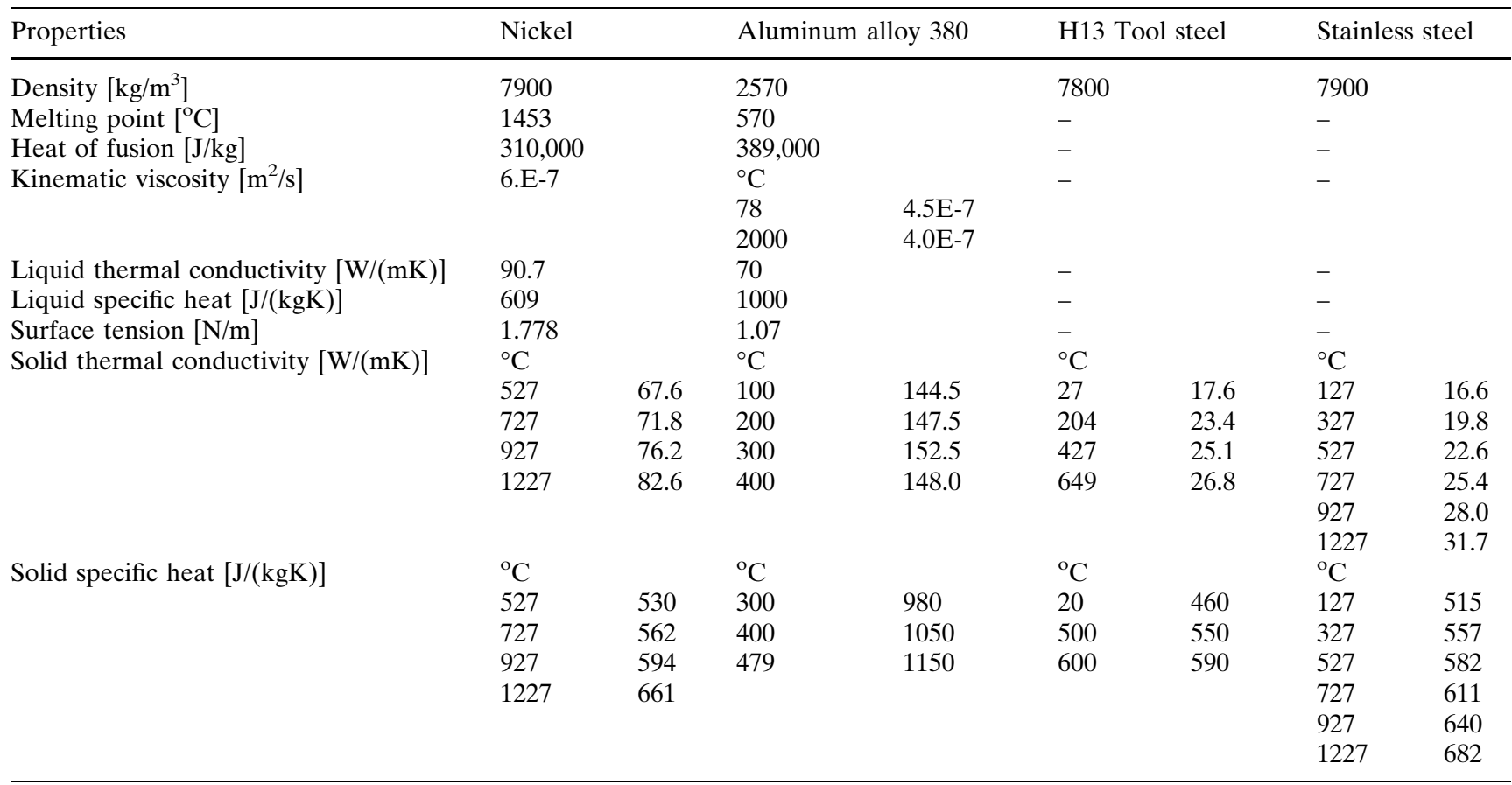

For substrate materials, the only properties needed are density, thermal conductivity, and specific heat

Cartesian coordinate system. We used uniform mesh sizes at all three dimensions. The substrate was extended far enough that its boundaries could be assumed to be at constant temperature. Numerical computations were performed on a Sun Ultra Enterprise 9.1 workstation. The typical CPU time to simulate an aluminum droplet impact was 3 days.

\section{Results and discussion}

Simulation of droplet deposition at low impact velocity

Figure 2 shows both photographs and computer generated images of the normal impact of a $3.92 \mathrm{~mm}$ diameter aluminum alloy 380 droplet with $3 \mathrm{~m} / \mathrm{s}$ velocity onto a $\mathrm{H} 13$ tool steel substrate at an initial temperature of $200{ }^{\circ} \mathrm{C}$. The initial droplet temperature was $630{ }^{\circ} \mathrm{C}$, about $60{ }^{\circ} \mathrm{C}$ above the melting point of the alloy. Average surface roughness $\left(R_{a}\right)$ was $0.5 \mu \mathrm{m}$. The time of each image, measured from the instant of first contact with the surface, was indicated. Computations were done on a domain that extended $8 \mathrm{~mm}$ in both $x$ and $y$ directions, $4 \mathrm{~mm}$ above the substrate in the $z$ direction and a depth of $2 \mathrm{~mm}$ into the substrate. The domain was meshed with 100 grid points in both $x$ and $y$ directions, and 32 grid points in the $z$ direction for both the droplet and the substrate. In the simulation, the contact resistance was calculated using Eq. (15) and it varied with time and position. Droplets impacted, spread into a thin disk, and solidified with a raised ridge around the periphery of the splat formed by the flattened droplet (see Fig. 2). Images from the simulation show good qualitative agreement with experimental photographs.

Figure 3 displays calculated temperature distribution inside the droplet, at the same times following impact as those in Fig. 2. Freezing of the metal began at the edges, which were in contact with the colder substrate, and blocked spreading of the liquid, contributing to the formation of raised edges. The growth of the solid layer, corresponding to portions of the droplet with $T \leq 570{ }^{\circ} \mathrm{C}$, can be clearly seen in these cross-sectional views.

To obtain a better quantitative comparison between simulations and experiments we measured the splat diameter $(D)$ from photographs at successive stages during droplet deformation and normalized it by the initial droplet diameter $\left(D_{0}\right)$ to calculate the spread factor $\left(\xi=D / D_{0}\right)$. Measured and predicted values of spread factor during the impact are shown in Fig. 4. Results from two sets of simulations are shown. The first was done with varying contact resistance, calculated from the local liquid pressure using Eq. (15), and it gave values of spread factor that lay within $10 \%$ of 


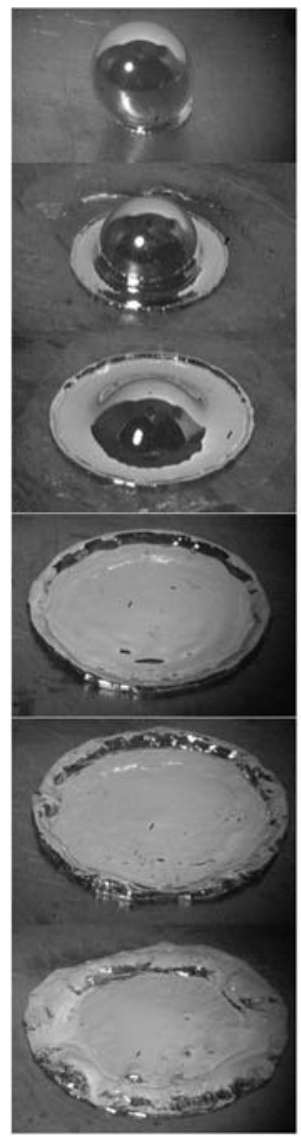

$0.1 \mathrm{~ms}$

$0.3 \mathrm{~m}$

$0.7 \mathrm{~ms}$

${ }^{200 m 5}$

${ }_{300 m}$

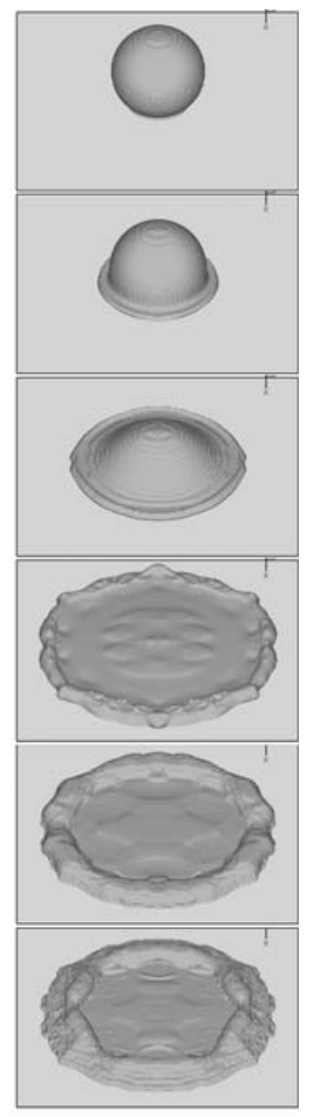

$5 \mathrm{~mm}$

\section{(a) Experiment}

$7.0 \mathrm{~ms}$

Fig. 2 Sequential impact of a $3.92 \mathrm{~mm}$ aluminum droplet on a tool steel substrate at $200{ }^{\circ} \mathrm{C}$ with $3 \mathrm{~m} / \mathrm{s}$ of impact velocity and $0.5 \mu \mathrm{m}$ of surface roughness

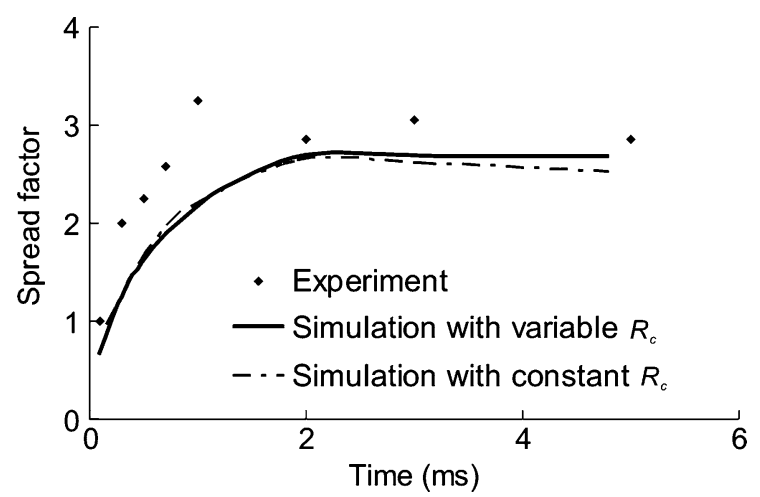

Fig. 4 Evolution of spread factor during the impact of an aluminum alloy droplet on a tool steel substrate initially at $200{ }^{\circ} \mathrm{C}$. The value of contact resistance for simulation with constant $R_{c}$ is $1.64 \times 10^{-6} \mathrm{~m}^{2} \mathrm{~K} / \mathrm{W}$

experimental results. For the second set of simulations a constant value of contact resistance was used, as done in previous investigations, where typically values of $R_{c i} \sim 10^{-6} \mathrm{~m}^{2} \mathrm{~K} / \mathrm{W}$ were used [12]. Trials showed that a value of $R_{c}=1.64 \times 10^{-6} \mathrm{~m}^{2} \mathrm{~K} / \mathrm{W}$ gave results that agreed closely with those obtained from the variable contact resistance trials. Of course, there was no way to determine this value a priori.

Figure 5 shows the radial variation of pressure, measured from the point of impact, at the interface between the substrate and droplet at three instants during impact. Figure 6 shows the distribution of thermal contact resistance, calculated from the liquid pressure. At $t=0.3 \mathrm{~ms}$ (measured from the instant of impact) the gauge pressure in the droplet centre was approximately $22 \mathrm{kPa}$, rising to $30 \mathrm{kPa}$ before dropping
Fig. 3 Simulated temperature distribution inside a $3.92 \mathrm{~mm}$ aluminum alloy droplet initially at $630{ }^{\circ} \mathrm{C}$ impacting with a velocity of $3 \mathrm{~m} / \mathrm{s}$ onto a tool steel substrate initially at $200{ }^{\circ} \mathrm{C}$

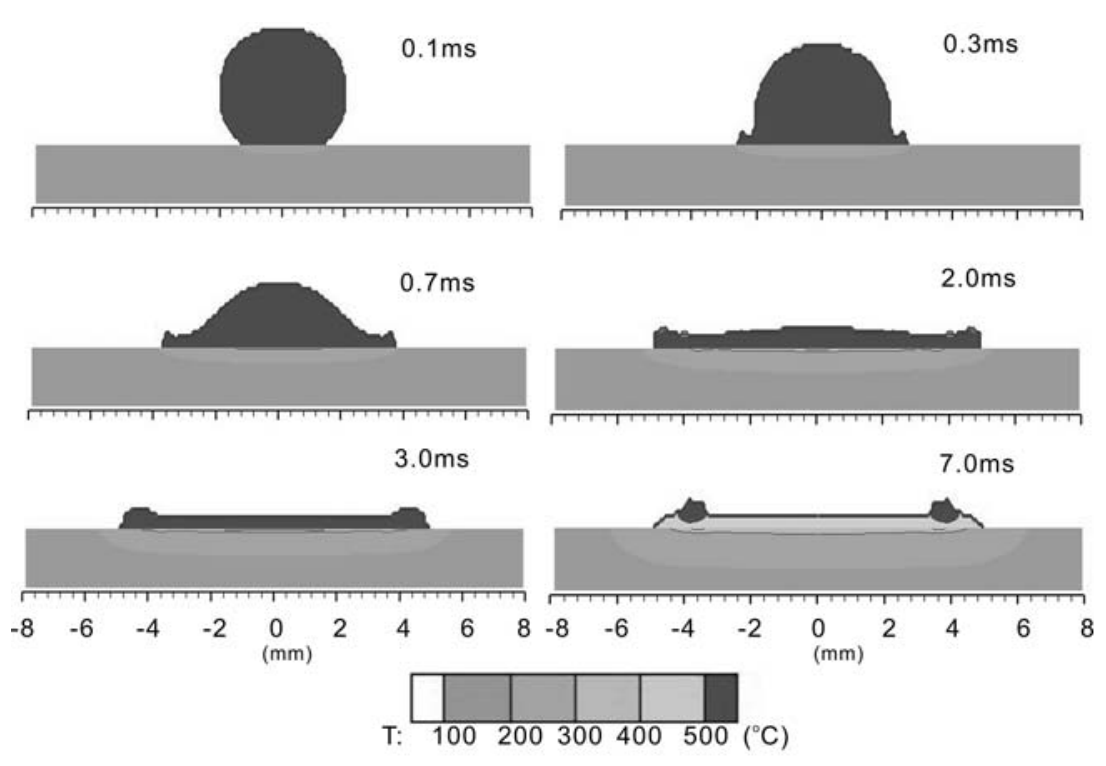




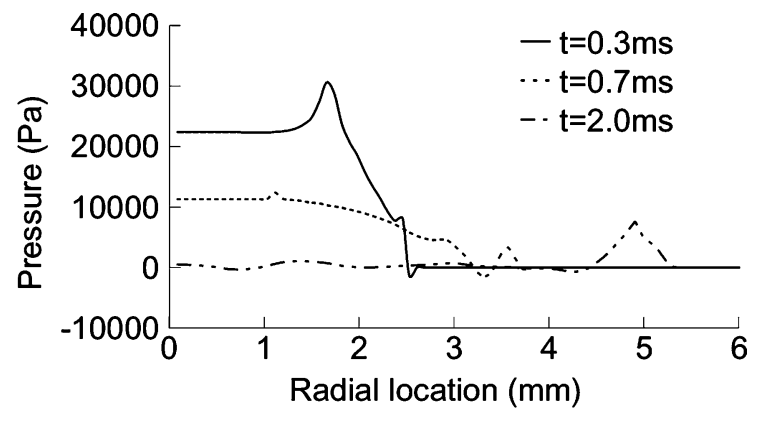

Fig. 5 Contact pressure distribution at the interface along one radius of the splat during the impact of aluminum alloy droplet on a tool steel substrate initially at $200{ }^{\circ} \mathrm{C}$, and the surface roughness is $0.5 \mu \mathrm{m}$

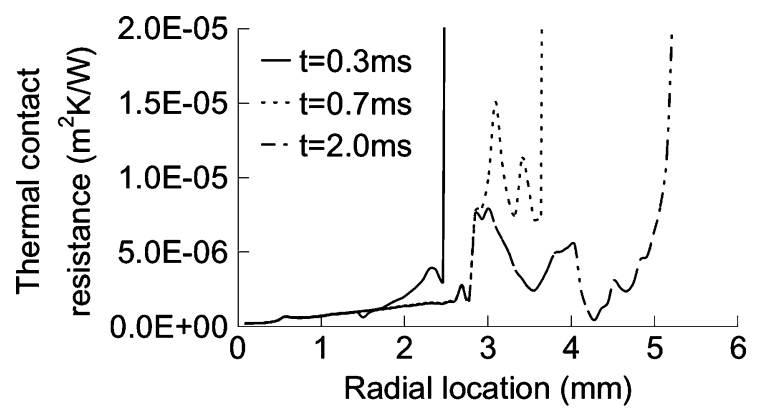

Fig. 6 Thermal contact resistance distribution at the interface along one radius of the splat during the impact of aluminum alloy droplet on a tool steel substrate initially at $200{ }^{\circ} \mathrm{C}$, and the surface roughness is $0.5 \mu \mathrm{m}$

off to zero at the droplet edge. The contact resistance followed an opposing trend, minimum at the center where pressure was highest, and increasing towards the edge. Beyond the edge of the splat, in empty computational cells, contact resistance was set to $10^{10} \mathrm{~m}^{2} \mathrm{~K} / \mathrm{W}$. As time advanced pressure in the droplet decreased (see Fig. 5), until it was completely dissipated by $t=2.0 \mathrm{~ms}$. However, contact resistance did not show a corresponding increase (Fig. 6) since we assumed that contact between the droplet and substrate, once established, would persist even when the pressure was relieved. Contact resistance values at any given point were calculated from the maximum local pressure encountered at that point.

Figure 7 shows the surface temperature variation of the $\mathrm{H} 13$ tool steel substrate under the splat center during the impact of an aluminum 380 alloy droplet with an initial temperature of $630{ }^{\circ} \mathrm{C}$. The tool steel substrate was initially at $200{ }^{\circ} \mathrm{C}$, and the surface roughness was $0.5 \mu \mathrm{m}$. The experimental results shown are temperature measurements by a thermocouple placed at the point of droplet impact. Results

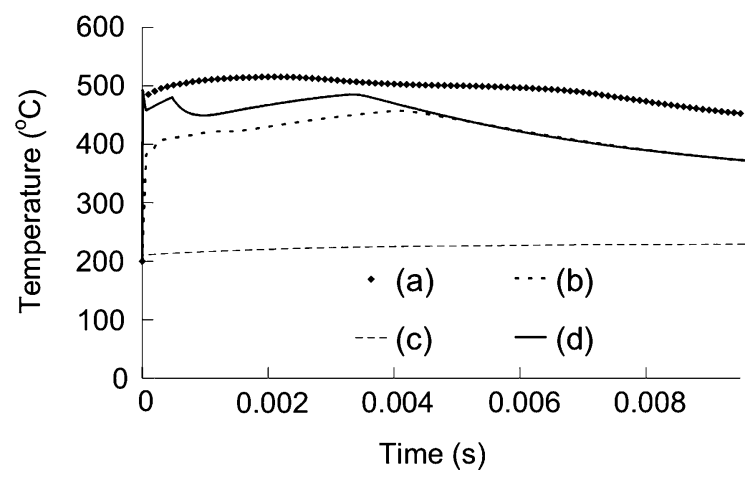

Fig. 7 Surface temperature histories of the steel substrate under the splat center during the impact of an aluminum alloy droplet. The substrate was initially at $200{ }^{\circ} \mathrm{C}$, surface roughness $0.5 \mu \mathrm{m}$. (a) Experimental measurements; (b) simulation with constant contact resistance $R_{c}=1.64 \times 10^{-6} \mathrm{~m}^{2} \mathrm{~K} / \mathrm{W} ;$ (c) simulation with insulated interface, where contact resistance $R_{c}=1.0 \times 10^{-4}$ $\mathrm{m}^{2} \mathrm{~K} / \mathrm{W}$; (d) simulation with variable thermal contact resistance

from simulations are shown for three different cases: for variable $R_{c}$, calculated from the model; with constant $R_{c}=1.64 \times 10^{-6} \mathrm{~m}^{2} \mathrm{~K} / \mathrm{W}$; and with $R_{c}=$ $10^{-4} \mathrm{~m}^{2} \mathrm{~K} / \mathrm{W}$. Experimentally, the surface temperature increased after the impact, reached a maximum of approximately $515{ }^{\circ} \mathrm{C}$ after $2.0 \mathrm{~ms}$ and then declined slowly. The simulation with variable contact resistance gave results that were closest to experiments. The discrepancy may have been due to the assumption of constant melting point made in the model: in reality aluminum alloy 380 has a liquidus temperature of $593{ }^{\circ} \mathrm{C}$ and solidus temperature of $538^{\circ} \mathrm{C}$, whereas in simulations a melting temperature of $570{ }^{\circ} \mathrm{C}$ was assumed. The simulation with a constant value of $R_{c}=1.64 \times 10^{-6} \mathrm{~m}^{2} \mathrm{~K} / \mathrm{W}$ gave even lower values of surface temperature, and when $R_{c}$ was set to $10^{-4} \mathrm{~m}^{2} \mathrm{~K} / \mathrm{W}$ there was barely any change in substrate temperature.

In experiments an array of thermocouples, spaced $1.15 \mathrm{~mm}$ apart, was placed under the impacting droplet [8]. Figure 8 a shows the variation of substrate temperature recorded by three thermocouples, located at radial positions, measured from the point of impact, $r=0,1.15 \mathrm{~mm}$ and $2.3 \mathrm{~mm}$. Immediately after impact the substrate temperature, initially at $200{ }^{\circ} \mathrm{C}$, increased to $500{ }^{\circ} \mathrm{C}$ in less than $0.3 \mathrm{~ms}$. The response of the second and third thermocouples lagged behind that of the first, as the edge of the spreading splat reached them. Figure $8 \mathrm{~b}$ shows the calculated temperature variation at the three thermocouple locations, calculated from the model using a variable contact resistance. The calculated variations are similar to those measured, though the maximum temperatures are slightly lower than those recorded in experiments. 

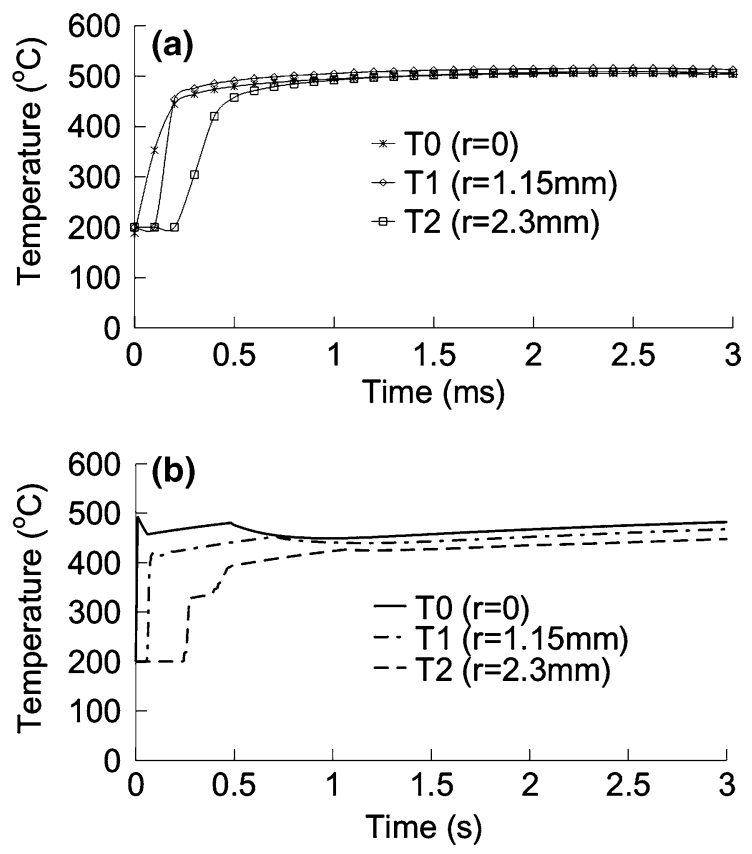

Fig. 8 (a) Experimental measurements and (b) simulation of temperature histories at three different radial locations of the splat

Simulation of plasma sprayed droplet at high impact velocity

Thermal contact resistance values between an impacting droplet and the substrate are of critical importance in simulations of molten metal droplet impact $[13,14]$. If the value is high, solidification of the particle is delayed until it has spread completely, and splashing is avoided so that circular, disk shaped splats are formed. If contact resistance is low the particle solidifies rapidly, leading to freezing around the edges of the spreading droplet, which obstructs flow and causes splashing. Typically, contact resistance values are not known, and serve as an adjustable parameter in models.

To determine if the model of contact resistance proposed here could be used to produce realistic simulation of thermal spray particle impact, we simulated the impact of plasma sprayed nickel powders, for which experimental results were available [1]. Based on measurements of particle properties using a DPV2000 diagnostic system (Tecnar Ltd., Montreal, Canada), the average velocity was $72 \mathrm{~m} / \mathrm{s}$, average particle diameter $50 \mu \mathrm{m}$, and average particle temperature $1654{ }^{\circ} \mathrm{C}$. Figure 9 shows the scanning electron microscope (SEM) images of splats formed by thermal plasma sprayed nickel particles on stainless steel substrate under these conditions with two different surface temperatures. On the surface initially at room
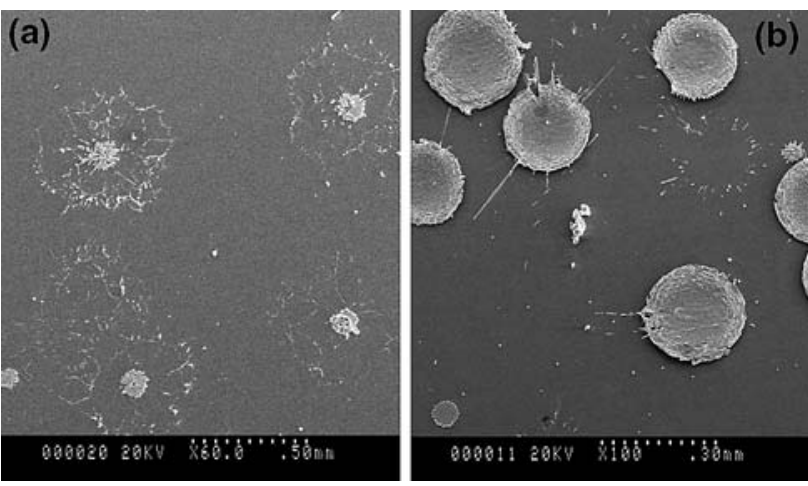

Fig. 9 The SEM image of thermal plasma sprayed nickel particles on a stainless steel substrate. The substrate initial temperature was (a) room temperature; (b) $360{ }^{\circ} \mathrm{C}$

temperature, splats were fragmented, consisting of a small, irregular core surrounded by debris from the break-up of the droplet (see Fig. 9a). On the heated surface, with $T_{w}=360{ }^{\circ} \mathrm{C}$, splats are, in general, disk shaped (see Fig. 9b).

Figure 10 shows successive stages during the simulated impact of a single liquid nickel droplet with a

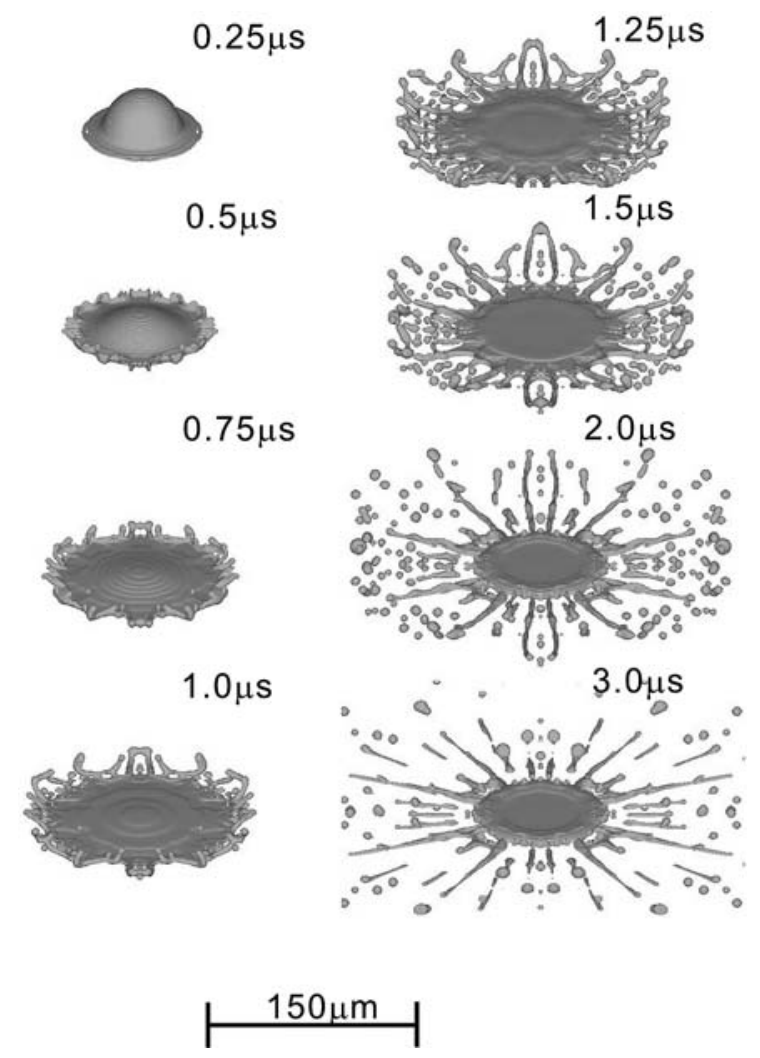

Fig. 10 Simulation view of sequence impact of a liquid nickel droplet with a diameter of $50 \mu \mathrm{m}$ initially traveling at $72 \mathrm{~m} / \mathrm{s}$ on a stainless steel substrate. The substrate initial temperature was $20{ }^{\circ} \mathrm{C}$, and the surface roughness was $0.5 \mu \mathrm{m}$ 
diameter of $50 \mu \mathrm{m}$ initially traveling at $72 \mathrm{~m} / \mathrm{s}$ impacting on a stainless steel substrate with surface roughness of $0.5 \mu \mathrm{m}$. The initial temperature of the droplet was $1654{ }^{\circ} \mathrm{C}$ and the substrate temperature was $20^{\circ} \mathrm{C}$. Thermal contact resistance was calculated with Eq. (15). The computational domain was $150 \mu \mathrm{m}$ wide, $150 \mu \mathrm{m}$ long, $60 \mu \mathrm{m}$ high and extended to a depth of $30 \mu \mathrm{m}$ into the substrate. The domain was meshed with 150 grid points in both $x$ and $y$ directions, 60 grid points in the $z$ direction above the substrate and 30 grid points into the substrate. As the droplet landed and spread, solidification started around the edges, disrupting flow of the spreading liquid sheet and forcing it to break up. By $t=3.0 \mu$ s the droplet had solidified, formed a splat surrounded by satellite droplets that that resembled those seen in photographs (see Fig. 9a).

Figure 11 shows the calculated pressure distributions under the droplet at $t=0.5,1.0$ and $1.5 \mu \mathrm{s}$, while Fig. 12 shows calculated contact resistance at the same times. Initially $(t=0.5 \mu \mathrm{s})$ there is a very high pressure region under the splat $(\sim 10 \mathrm{MPa})$, which produces low contact resistance. The pressure decays to zero very rapidly (by $t=1.5 \mu \mathrm{s}$ ), but the contact resistance near

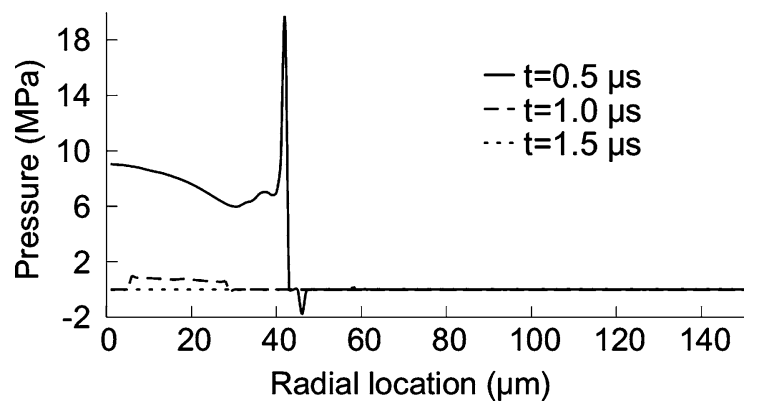

Fig. 11 Contact pressure distribution at the interface along one radius of the splat during the impact of nickel droplet on a tool steel substrate initially at $20^{\circ} \mathrm{C}$, and the surface roughness was $0.5 \mu \mathrm{m}$

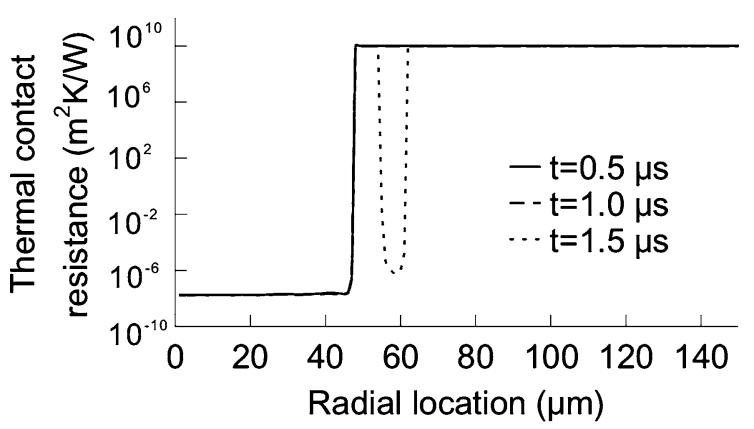

Fig. 12 Thermal contact resistance distribution at the interface along one radius of the splat during the impact of nickel droplet on a tool steel substrate initially at $20^{\circ} \mathrm{C}$, and the surface roughness was $0.5 \mu \mathrm{m}$ the centre of the splat remains low because of the ever high pressure at the center of the splat. As the edges of the splat fragment the contact resistance alternated between low and high values, since empty cells were postulated to have a contact resistance of $1010 \mathrm{~m}^{2} \mathrm{~K} / \mathrm{W}$.

Increasing the substrate temperature to $360{ }^{\circ} \mathrm{C}$ produced relatively little change in the splat shape. Figure 13a shows the final splat shape for a $50 \mu \mathrm{m}$ diameter nickel particle impacting under the conditions given above, where the dynamics of impact are shown in Fig. 10. Raising the substrate temperature to $360{ }^{\circ} \mathrm{C}$ delayed the onset of solidification slightly, so that the central splat was slightly larger (see Fig. 10b), and splashing was somewhat reduced. However, the change was relatively minor. The change in splat shape seen in the photographs of Fig. 9 cannot be explained by the change in surface temperature alone.

It has previously been shown that heating a steel plate produces an oxide layer on its surface, which may result in an increase in contact resistance between the droplet and substrate [1]. This increase would not be predicted by the contact resistance model, which accounts solely for surface roughness. It can be represented, though, by a constant thermal resistance added to the previously calculated contact resistance value. Figure 14a shows the final splat shape for a $50 \mu \mathrm{m}$ nickel particle impacting with $72 \mathrm{~m} / \mathrm{s}$ velocity on a steel surface at $360{ }^{\circ} \mathrm{C}$. A constant value of $10^{-7} \mathrm{~m}^{2} \mathrm{~K} / \mathrm{W}$ was added to thermal contact resistances calculated from Eq. (15). Break-up of the splat is greatly reduced, with a large disc-shaped splat, surrounded by a few satellite droplets. If the constant contact resistance was increased to $2 \times 10^{-7} \mathrm{~m}^{2} \mathrm{~K} / \mathrm{W}$, splat break-up was almost completely eliminated, producing a disc-shaped splat with a few small fingers radiating from it, which resemble the photographs of Fig. 9 b.

\section{Summary and conclusions}

An analytical model to calculate thermal contact resistance between a molten metal droplet and a rough, solid surface can be incorporated into a numerical model of droplet impact to calculate local thermal contact resistance at each point under an impacting droplet. A value of the thermal contact resistance does not then have to be provided to the model; the only inputs are the substrate roughness and thermal conductivity.

The numerical model was able to accurately predict the impact dynamics of $4 \mathrm{~mm}$ diameter aluminium alloy droplets landing on a tool steel plate with $3 \mathrm{~m} / \mathrm{s}$ velocity, and the substrate temperature variation under 
Fig. 13 Simulation view of the final splat shapes of a liquid nickel droplet with a diameter of $50 \mu \mathrm{m}$ initially traveling at $72 \mathrm{~m} / \mathrm{s}$ on a stainless steel substrate. The surface roughness was $0.5 \mu \mathrm{m}$. The substrate initial temperature was (a) $20{ }^{\circ} \mathrm{C}$, and (b) $360{ }^{\circ} \mathrm{C}$ (a)

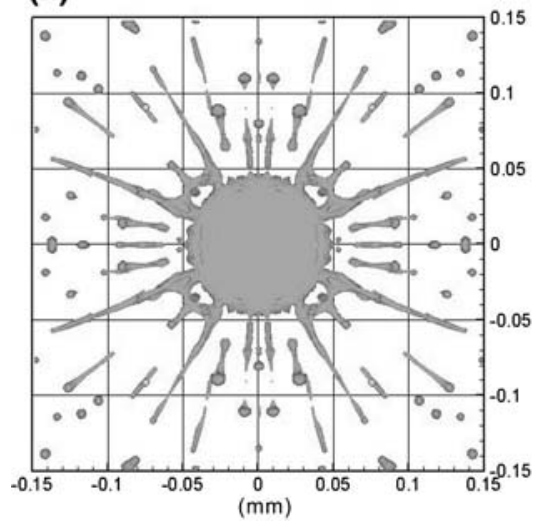

(b)

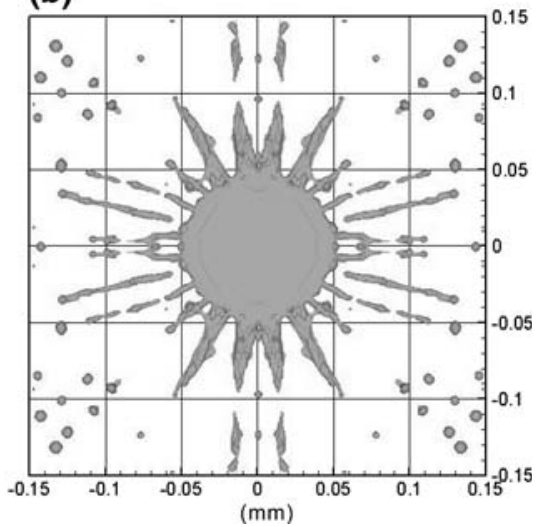

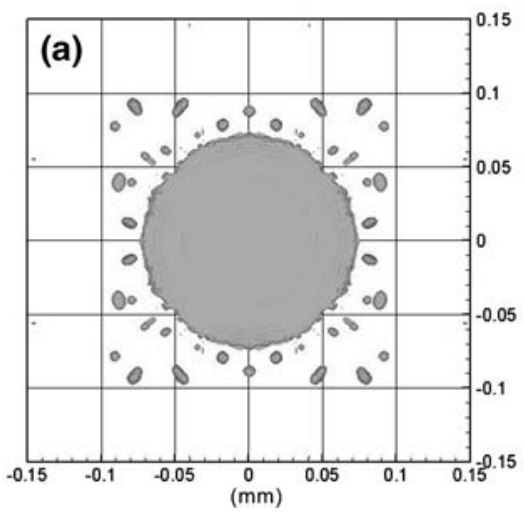

Fig. 14 Simulation view of the final splat shape of a liquid nickel droplet with a diameter of $50 \mu \mathrm{m}$ initially traveling at $72 \mathrm{~m} / \mathrm{s}$ on a stainless steel substrate. The surface roughness was $0.5 \mu \mathrm{m}$. The substrate initial temperature was $360^{\circ} \mathrm{C}$. The contact resistance

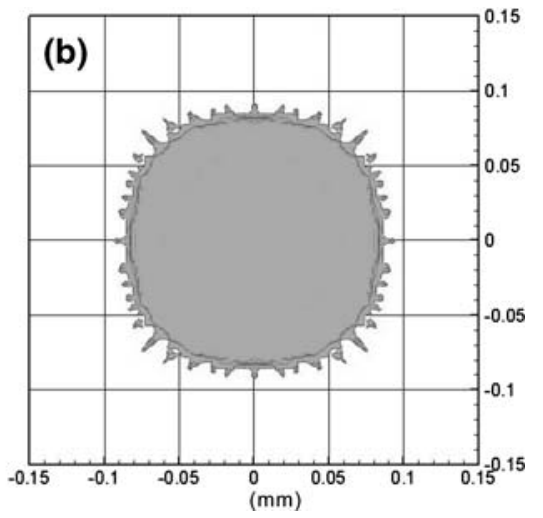

scheme was modified so that in: (a) a constant $R_{c}=1.0 \times 10^{-7}$ $\mathrm{m}^{2} \mathrm{~K} / \mathrm{W}$ was added to the variable thermal contact resistance calculated from Eq. (15); (b) a constant thermal contact resistance $R_{c}=2.0 \times 10^{-7} \mathrm{~m}^{2} \mathrm{~K} / \mathrm{W}$ was used

3. Bussmann M, Mostaghimi J, Chandra S (1999) Phys Fluids 11:1406

4. Pasandideh-Fard M, Chandra S, Mostaghimi J (2002) Int J Heat Mass Transfer 45:2229

5. Mikic BB (1974) Int J Heat Mass Transfer 17:205

6. Wang G-X, Matthys EF (2002) Int J Heat Mass Transfer 45:4967

7. Hong FJ, Qiu H-H (2005) Numer Heat Transfer 48:987

8. Heichal Y, Chandra S (2005) J Heat Transfer 127:1269

9. Cheng SX, Li T, Chandra S (2005) J Mater Process Technol 159:295

10. Chandra S, Avedisian CT (1990) Phys Fluids A2:1525

11. Timsit RS (1982) J Wear 83:129

12. Aziz S, Chandra S (2000) Int J Heat Mass Transfer 43:2841

13. Mehdizadeh Z, Raessi M, Chandra S, Mostaghimi J (2004) J Heat Transfer 126:445

14. Pasandideh-Fard M, Pershin V, Chandra S, Mostaghimi J (2002) J Therm Spray Technol 11:206

\section{References}

1. Pershin V, Lufitha M, Chandra S, Mostaghimi J (2003) Therm Spray Technol 12:370

2. Ghafouri-Azar R, Mostaghimi J, Chandra S, Charmchi M (2003) J Therm Spray Technol 12:53 